\title{
Pulmonary vein parameters are similar or better predictors than left atrial diameter for paroxysmal atrial fibrillation after cryoablation
}

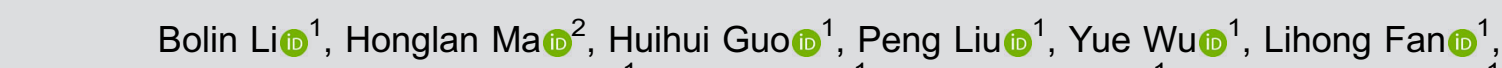 \\ Yumeng Cao (i) ${ }^{1}$, Zhijie Jian (ii) ${ }^{1}$, Chaofeng Sun (ii) ${ }^{1}$, and Hongbing $\mathrm{Li}_{\text {(i) }}{ }^{1}$ \\ ${ }^{1}$ Department of Cardiovascular Medicine, The First Affiliated Hospital of Xi'an Jiaotong University, Xi'an, Shaanxi, China \\ ${ }^{2}$ Department of Cardiovascular Medicine, The First Affiliated Hospital of Xi'an Medical College, Xi'an, Shaanxi, China
}

\begin{abstract}
Left atrial diameter (LAD) has been considered an independent risk factor for atrial fibrillation (AF) relapse after pulmonary vein isolation $(\mathrm{PVI})$. However, whether LAD or other factors are more predictive of late recurrence in patients with paroxysmal AF remains unclear. We aimed to evaluate the value of pulmonary vein (PV) parameters for predicting AF relapse 1 year after patients underwent cryoablation for paroxysmal AF. Ninety-seven patients with paroxysmal AF who underwent PVI successfully were included. PV parameters were measured through computed tomography scans prior to PVI. A total of 28 patients had recurrence of $A F$ at one-year follow-up. The impact of several variables on recurrence was evaluated in multivariate analyses. $L A D$ and the time from first diagnosis of $A F$ to ablation maintained its significance in predicting the relapse of $A F$ after relevant adjustments in multivariate analysis. When major diameter of right inferior pulmonary vein (RIPV) (net reclassification improvement (NRI) $0.179, \mathrm{Cl}=0.031-0.326, \mathrm{P}<0.05)$ and cross-sectional area (CSA) of RIPV (NRI: $0.122, \mathrm{Cl}=0.004-0.240$, $\mathrm{P}<0.05)$ entered the AF risk model separately, the added predictive capacity was large. The accuracy of the two parameters in predicting recurrence of AF were not inferior (AUC: 0.665 and 0.659 , respectively) to echocardiographic LAD (AUC: 0.663 ). The inclusion of either RIPV major diameter or CSA of RIPV in the model increased the C-index ( 0.766 and 0.758 , respectively). We concluded that major diameter of RIPV had predictive capacity similar to or even better than that of LAD for predicting AF relapse after cryoablation PVI.
\end{abstract}

Key words: Atrial fibrillation; Cryoablation; Right inferior pulmonary vein; Pulmonary vein parameter

\section{Introduction}

Atrial fibrillation (AF) is a fairly common arrhythmia worldwide, with an estimated 33 million people suffering from this condition (1). Pulmonary vein isolation (PVI), according to guidelines (2), is recommended for symptomatic, drug-refractory, paroxysmal AF. As cryoablation has recently been introduced in patients with paroxysmal $A F$, this procedure may allow a simpler approach to electrically isolate the pulmonary vein (PV) from the left atrium (LA) to prevent recurrent AF. Short-to-midterm studies employing cryoballoons have shown recurrence rates after ablation ranging from 67 to $83.6 \%$ for patients with drug-refractory paroxysmal AF (3-5). The reason for AF relapse is not completely understood but is related to restored PV conduction $(6,7)$. It is accepted that reestablishment of conduction is mainly due to gaps between the ablated lesions or less-than-optimal lesion depth, which has a considerable effect on recurrence (8). The extent to which anatomic parameters of the PV might predict electrical reconnection has been shown in a few studies (9-11).

Most research documented the long-term prognostic value of LA diameter or morphology deformities. In fact, outcomes of ablation are highly dependent on atrial disease stage, and the timing of chamber dilation may happen later than regional deformities (12). However, there is no consensus on the influence of increased PV ostium diameter, size, or roundness on the clinical efficacy of cryothermal PVI techniques. The goal of our study was to assess the value of these PV parameters for predicting the risk of recurrence of paroxysmal $A F$ in patients who underwent cryoablation. 


\section{Material and Methods}

For this prospective observational study, we enrolled 100 patients (October 2014 to June 2017) with symptomatic paroxysmal AF who had or had not taken antiarrhythmic drugs owing to refusal of chronic drug therapy. This study of cryoballoon ablation versus radiofrequency ablation for paroxysmal AF was performed in the Cardiology Department of the First Affiliated Hospital of Xi'an JiaoTong University and registered at the Chinese Clinical Trial Registry (ChiCTR1800016314). As per the 2014 American Heart Association/American College of Cardiology/Heart Rhythm Society (AHA/ACC/HRS) AF guidelines (13), paroxysmal $A F$ was defined as $A F$ that terminated spontaneously or with intervention within 7 days of onset and that may recur with variable frequency. Medical histories were obtained during clinic visits, and all medical records including electrocardiograms (ECGs) and Holter ECG recordings showing AF episodes were reviewed. The study protocol (conforming to the ethical guidelines of the Declaration of Helsinki) was approved by the institutional ethics committee. We excluded patients who presented with moderate-to-severe valvular stenosis or regurgitation, acute reversible causes of $A F$, previous $A F$ ablation procedures, myocardial infarction that occurred within 3 months, severe respiratory insufficiency, left atrial thrombi, left ventricular ejection fraction $<40 \%$, anatomic variations of the number of PV ostia, pregnancy, or New York Heart Association class III or IV heart disease.

For each patient, all images were obtained with 256slice computed tomography (CT) scan (Brilliance-i CT; Philips Healthcare, USA) following intravenous injection of non-ionic contrast medium (lohexol Injection, $100 \mathrm{~mL}$ at $5 \mathrm{~mL} / \mathrm{s}, 128 \mathrm{~mm} \times 0.625 \mathrm{~mm}$ collimation). Images were obtained from the level of the diaphragm to the aortic arch scanning in high-pitch spiral acquisition mode during a single breath-hold. Prospective electrocardiographic gating was considered to eliminate cardiac motion artifact. Image reconstruction was accomplished by a post-processing workstation using $2 \mathrm{D}$ viewing modes and $3 \mathrm{D}$ reconstruction. After $3 \mathrm{D}$ and $2 \mathrm{D}$ images were acquired, the right and left PVs were identified, and the maximum and minimum diameters of the PV ostia were measured in optimal images. The form of the PV ostia was identified by using a venous ostium index, which was calculated by dividing the minor axis by the major axis for each PV. The cross-sectional area (CSA) was calculated as $p \times$ (major axis / $2 \times$ (minor axis / 2) for each PV (14).

Cryoablation was performed under local anesthesia by one of two operators. Briefly, a bolus of heparin was administered intravenously preceding transseptal puncture to maintain an activated clotting time between 250 to $350 \mathrm{~s}$. A deflectable transseptal guiding introducer (8 F, $2.6 \mathrm{~mm}$, St. Jude Medical, USA) was inserted via free-hand technique into the right subclavian vein and placed in the coronary sinus to guide the transseptal needle $(1.3 / 0.5 \mathrm{~mm}$, St. Jude Medical) to successfully puncture the atrial septum. Then, a second-generation cryoballoon (Medtronic CryoCath LP, Canada) was introduced into the LA. Once the balloon was expanded in the $\mathrm{PV}$, occlusion was assessed with $50 \%$ diluted contrast injection. Subsequently, cryoenergy was delivered to each PV for two freezing cycles of less than $180 \mathrm{~s}$. We began to operate more often in the left superior pulmonary vein (LSPV) than in the left inferior (LIPV), right superior (RSPV), or right inferior pulmonary vein (RIPV). Finally, $\mathrm{PV}$ conduction was re-evaluated with the Achieve catheter (Medtronic Inc., USA). Successful PVI was considered isolation of all the $\mathrm{PV}$ potentials recorded from the Achieve catheter. For perioperative AF without conversion to sinus rhythm, direct current cardioversion was performed.

Evidence of atrial thrombus formation was evaluated by transesophageal echocardiography. M-mode, 2-dimensional, and Doppler echocardiography were performed and analyzed by 2 cardiologists in accordance with the American Society of Echocardiography guidelines (15) For each patient, laboratory tests were conducted after fasting for $12 \mathrm{~h}$. All patients were informed of the necessity and risks of cryoballoon ablation, and they all provided written informed consent before the ablation procedure.

After successful PVI, the patients were sent back to the ward and monitored for $\geqslant 24 \mathrm{~h}$. Rivaroxaban or dabigatran were administered to prevent thrombus formation, and amiodarone was prescribed for at least 3 months post-procedure. Patients were followed up at 3, 6, 9, and 12 or more months and at any time they felt uncomfortable after the ablation. ECGs, 24-h Holter ECG recordings, and echocardiography were performed at each scheduled follow-up visit. Recurrent AF was considered if symptomatic or asymptomatic AF lasting for more than $30 \mathrm{~s}$ after 3 months blanking period was documented on an ECG or Holter study.

Data are reported as means $\pm S D$ or median and interquartile range for continuous variables and percentages for categorical variables. Continuous variables that had a normal distribution were evaluated using Student's $t$-test, whereas the Mann-Whitney $U$ test was used for non-normally distributed data. Categorical variables and frequencies were compared with the chi-squared test. The baseline clinical variables between patients with or without AF relapse during follow-up were selected for the multivariate analysis. The RIPV parameters including major diameter, minor diameter, and CSA that differed significantly between patients with and without recurrence of AF were tested one at a time by the $\mathrm{C}$ statistic. The improvement of discrimination when one RIPV risk marker was added to the risk model was assessed using the C-index and integrated discrimination improvement (IDI) (16), and reclassification was estimated by using continuous net reclassification improvement (NRI). The cumulative proportional probability of AF recurrence for continuous variables was studied using Kaplan-Meier survival analysis 
with the log-rank test, and the optimal cutoff point was determined using receiver operating characteristic curves. SPSS version 22.0.0 (IBM, USA) and $\mathrm{R}$ version 3.4.4 (https://www.r-project.org) were used for statistical analyses, and a two-sided $P$ value of $<0.05$ was considered statistically significant.

\section{Results}

Three patients receiving further radiofrequency ablation for PV conduction recorded from the Achieve catheter were excluded. Thus, ninety-seven patients with paroxysmal AF were included in the study and completed a 12month follow-up. Ultimately, $28(28.9 \%)$ patients with AF relapse were classified into one group, and the remaining 69 patients without recurrence were included in the other group (Table 1). The RIPV parameters (major diameter, minor diameter, and CSA) and left atrial diameter (LAD) were larger in patients with recurrence than in those without recurrent AF (Table 1 and Figure 1). When baseline clinical variables between the two groups (Table 1) were included in the Cox multivariate clinical hazards model, LAD (HR: 1.213, Cl: 1.051-1.400, $\mathrm{P}<0.01$ ) and the time from first diagnosis of AF to ablation (HR: 1.134, $\mathrm{Cl}: 1.017-1.265, \mathrm{P}<0.05)$ were determined to be independent predictors of AF relapse (Figure 2A).

The reclassification of patients with vs without the occurrence of AF improved significantly when the major LAD (NRI: 0.179, Cl: 0.031-0.326, $\mathrm{P}<0.05$ ) and CSA of
RIPV (NRI: 0.122, Cl: 0.004-0.240, P <0.05) were added to the model separately. The IDI of patients was significant when the major diameter of RIPV (IDI: 0.071, Cl: 0.003$0.138, \mathrm{P}<0.05)$ was included in the AF prediction model. Including the two parameters above $(0.766$ and 0.758 , respectively) in the model increased the $\mathrm{C}$-index moderately (Table 2). Information on the calibration of the models has been provided in Supplementary Figure S1. Based upon these findings, a nomogram was configured (Figure 2B).

Kaplan-Meier curves were used to illustrate the cumulative proportional probability of AF recurrence (values of major and minor diameters, and CSA of the RIPV less than or equal to the chosen cutoff points vs values greater than the chosen cutoff points) in Figure $3 \mathrm{~A}$. The accuracies of LAD, major and minor diameters of the RIPV, and CSA of the RIPV for predicting AF relapse are shown as the areas under the receiver operating characteristic curves (Figure 3B).

\section{Discussion}

The main finding of the present study was that among the studied PV parameters, RIPV parameters were the strongest independent predictors of long-term risk of recurrent AF after cryoablation. These parameters, especially the major diameter of the RIPV, predicted AF relapse as well as or even better than LAD, and improved the accuracy of the AF prediction model.

PVs play an essential role in the pathophysiology of AF. Approximately $90 \%$ of PVs contain atrial myocardial

Table 1. Baseline patient characteristics.

\begin{tabular}{|c|c|c|c|}
\hline Characteristic & No AF $(n=69)$ & AF $(n=28)$ & $\mathrm{P}$ \\
\hline Time from diagnosis to ablation (years) & $4.1 \pm 4.5$ & $6.1 \pm 6.3$ & 0.094 \\
\hline Age (years) & $61.2 \pm 10.4$ & $64.0 \pm 7.6$ & 0.148 \\
\hline Gender (male) & $38(55 \%)$ & $15(54 \%)$ & 0.893 \\
\hline BMI & $24.6 \pm 2.1$ & $25.0 \pm 2.6$ & 0.465 \\
\hline Alcohol use & $6(9 \%)$ & $3(11 \%)$ & 0.715 \\
\hline Smoking & $15(22 \%)$ & $7(25 \%)$ & 0.728 \\
\hline Hypertension & $35(51 \%)$ & $13(46 \%)$ & 0.701 \\
\hline Diabetes mellitus & $9(13 \%)$ & $5(18 \%)$ & 0.537 \\
\hline $\mathrm{CHD}$ & $21(30 \%)$ & $13(46 \%)$ & 0.135 \\
\hline $\mathrm{CHA}_{2} \mathrm{DS}_{2}-\mathrm{VAS}_{\mathrm{C}}$ score $(\geqslant 2)$ & $36(52 \%)$ & $20(71 \%)$ & 0.091 \\
\hline NT-proBNP (pg/mL) & $306(161-1355)$ & $337(79-829)$ & 0.238 \\
\hline LAD (mm) & $35.1 \pm 4.1$ & $37.6 \pm 4.2$ & $<0.05$ \\
\hline Septal wall thickness $(\mathrm{mm})$ & $8.56 \pm 1.14$ & $8.56 \pm 1.31$ & 0.985 \\
\hline LVEDD (mm) & $50.7 \pm 6.6$ & $49.9 \pm 3.7$ & 0.571 \\
\hline LVESD (mm) & $31.1 \pm 5.5$ & $31.6 \pm 3.5$ & 0.647 \\
\hline LVEF (\%) & $66 \pm 5$ & $65 \pm 6$ & 0.426 \\
\hline
\end{tabular}

Data are reported as means $\pm S D$, percentage, or median and interquartile range. AF: atrial fibrillation; BMI: body mass index; CHD: coronary heart disease; NT-proBNP: N-terminal pro brain natriuretic peptide; LAD: left atrial diameter; LVEDD: left ventricular end-diastolic diameter; LVESD: left ventricular end-systolic diameter; LVEF: left ventricular ejection fraction. Student's $t$-test, Mann-Whitney U test, or chi-squared test were used to compare the two groups. 
A

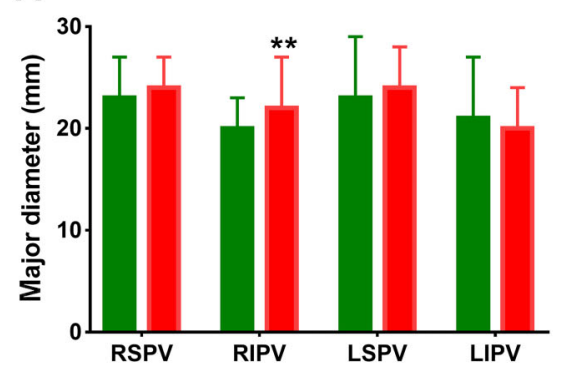

C

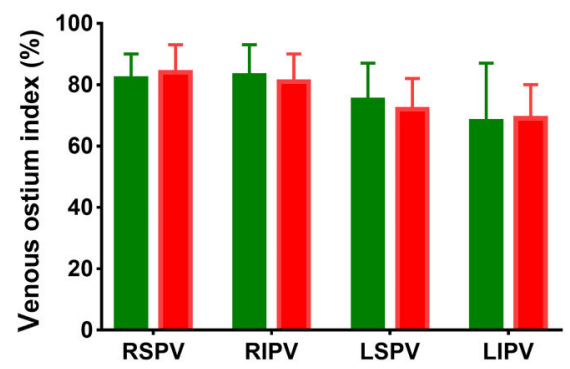

B

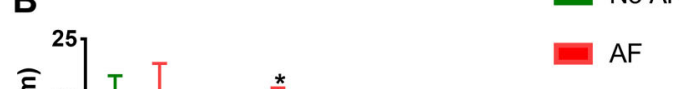

D

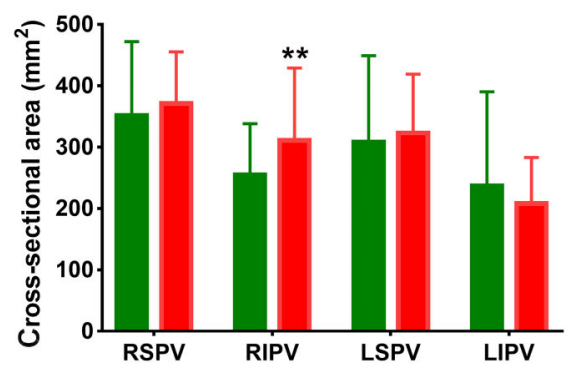

Figure 1. Parameters of pulmonary veins. AF: atrial fibrillation; RSPV: right superior pulmonary vein; RIPV: right inferior pulmonary vein; LSPV: left superior pulmonary vein; LIPV: left inferior pulmonary vein. Data are reported as means $\pm S D{ }^{*} P<0.05$, ${ }^{* *} P<0.01$ between groups (Student's $t$-test or Mann-Whitney $U$ test).

tissue (17), and the myocardium in PVs is often discontinuous and fibrotic. Abnormalities in the electrical activity in PVs is closely related to AF in many patients (18). Recurrent AF has generally been attributed to the recovery of electrical function after PVI $(6,7)$. Larger PVs with more discontinuous and fibrotic atrial myocardium may have a greater frequency of electrophysiological abnormalities, may be more difficult to isolate, and may be more likely to undergo electrical reconnection than those without these abnormalities.

However, there is no consensus regarding which PV parameters are most strongly associated with AF recurrence in patients undergoing AF ablation. Guler et al. (10) showed that increased RSPV size was a predictor of recurrence. Tsyganov et al. also found that a larger LIPV size was associated with worse long-term outcomes but was not a major risk factor for short- or long-term failure after PVI (9). In the Multicenter Sustained Treatment of Paroxysmal Atrial Fibrillation (STOP-AF) study, there was no correlation between changes in PV parameters and early or late AF recurrence (19). The prognostic value of RIPV parameters were validated in the patients with $A F$ receiving cryoablation in our study. The finding was partially consistent with the research conducted by Shimamoto et al. (20). Their study showed that the value of increased RIPV size for predicting AF recurrence in patients who underwent RF ablation was the most pronounced among PVs ostial area.
Isolation of the RIPV is often thought to be more challenging than isolation of other PV branches mainly due to proximity to the transseptal puncture site and the location of the RIPV itself. Incomplete myocyte damage and local tissue edema may contribute to the appearance of conduction block in patients who may later develop recurrent conduction after myocardial healing. Relapsed $\mathrm{AF}$ is highly correlated with lack of scarring in the inferior portion of the RIPV (82\%) (21). Studies also confirmed that the majority of new triggers arose from the RIPV, which most commonly reablated during a repeat ablation (22-24).

Increased LAD is widely accepted as a predictor of recurrent $A F(17,25-27)$. Tsao et al. confirmed that the diameters of the PV ostia and LA were increased in patients with AF compared to those without AF (28). Chen et al. (29) have suggested that increased LAD may not be a main risk factor for anatomical remodeling. Research suggested patients with paroxysmal AF had less fibrosis and smaller LA size compared with persistent AF (30). Atrial remodeling of the left atrium in paroxysmal AF was in the early stages, and asymmetrical deformities may occur before chamber dilation (12). Thus, LAD may not have been sensitive enough to reflect an early stage of anatomical remodeling. Potential importance of LA asymmetry index (31), LA sphericity (32), or 3D geometrical models from the segmentation of the LA blood pool (33) were also studied in predicting AF ablation, but clinical 


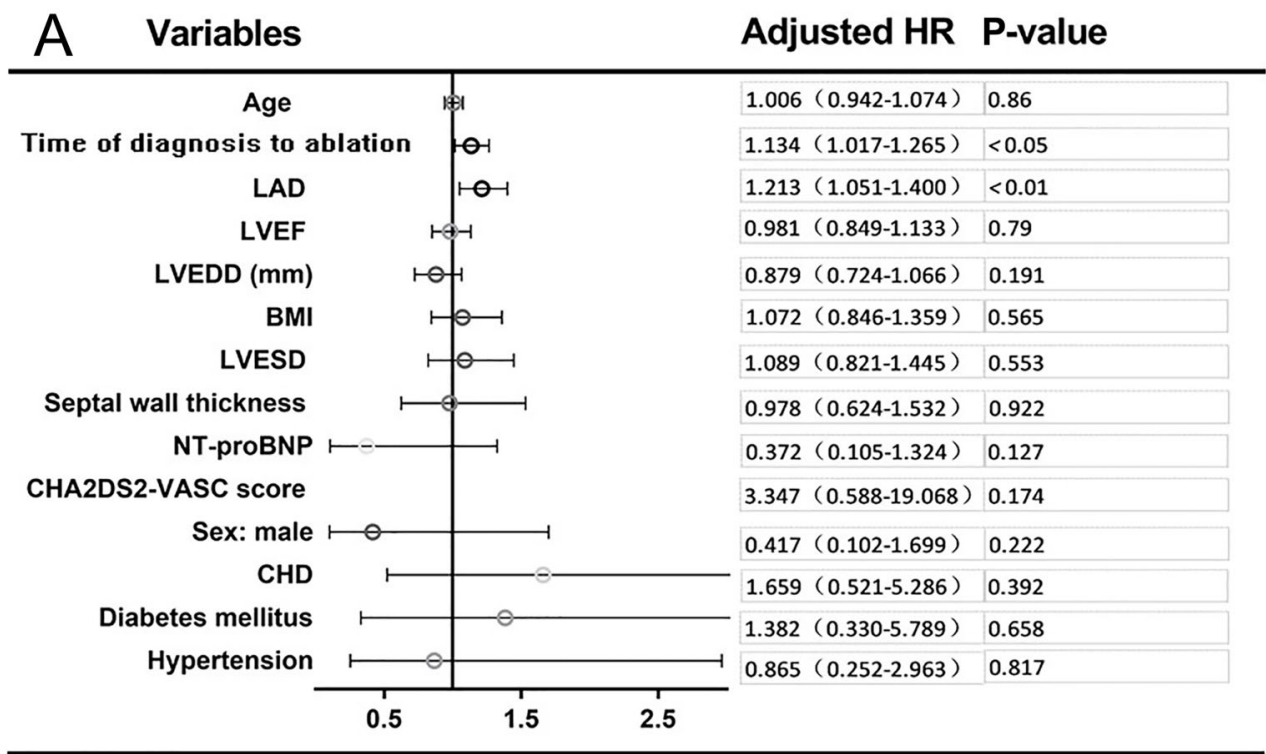

B $\begin{aligned} & \text { B } \\ & \text { Points }\end{aligned}$
History
LADc

Figure 2. A, Adjusted hazard ratio and $95 \%$ confidence intervals for each baseline clinical variable between patients with and without atrial fibrillation recurrence during follow-up. Cl: confidence interval; HR: hazard ratio; LAD: left atrial diameter; BMI: body mass index; CHD: coronary heart disease; NT-proBNP: N-terminal pro brain natriuretic peptide; LVEDD: left ventricular end-diastolic diameter; LVESD: left ventricular end-systolic diameter; LVEF: left ventricular ejection fraction. B, Instruction for using the nomogram. Draw a line perpendicular from the corresponding axis of each risk factor until it reaches the top line labeled "Points." Sum up the number of points for all risk factors then draw a line descending from the axis labeled "Total Points" until it intercepts each of the survival axes to determine 3- and 12-month relapse-free survival rates. Continuous variables such as LAD and major diameter of right inferior pulmonary vein were converted to categorical variables (LADc and RIPV1c), 0 for no and 1 for yes.

series can be difficult. Measurements of LA segments, such as the diameter and CSA of ostium of PV parameters, could provide an easy and generally available way of predicting recurrence.
This study was limited by its relatively small sample size and because it was a single-center study involving a highly selected population. The lack of continuous monitoring might have led to underestimation of the recurrence 
Table 2. Pulmonary vein parameters for the prediction of improvement in integrated discrimination (IDI) and net reclassification (NRI).

\begin{tabular}{lccccc}
\hline Variable & C-index & IDI $(95 \% \mathrm{Cl})$ & $\mathrm{P}$ & $\mathrm{NRI}(95 \% \mathrm{Cl})$ & $\mathrm{P}$ \\
\hline Established model & $0.691(0.460-0.923)$ & Reference & & Reference \\
+ RIPV major D & $0.766(0.534-0.997)$ & $0.071(0.003-0.138)$ & $<0.05$ & $0.179(0.031-0.326)$ & $<0.05$ \\
+ RIPV minor D & $0.736(0.504-0.968)$ & $0.022(-0.014-0.0585)$ & 0.230 & $0.050(-0.024-0.125)$ & 0.185 \\
+ RIPV-CSA & $0.758(0.526-0.990)$ & $0.049(-0.006-0.105)$ & 0.081 & $0.122(0.004-0.240)$ & $<0.05$ \\
\hline
\end{tabular}

The baseline variables that differed significantly between patients with and without atrial fibrillation recurrence, such as time from diagnosis to ablation and left atrial diameter, were included in the established model. Right inferior pulmonary vein (RIPV) major diameter (D), minor D, and cross-sectional area (CSA) were added to this model as continuous variables. Cl: confidence interval.
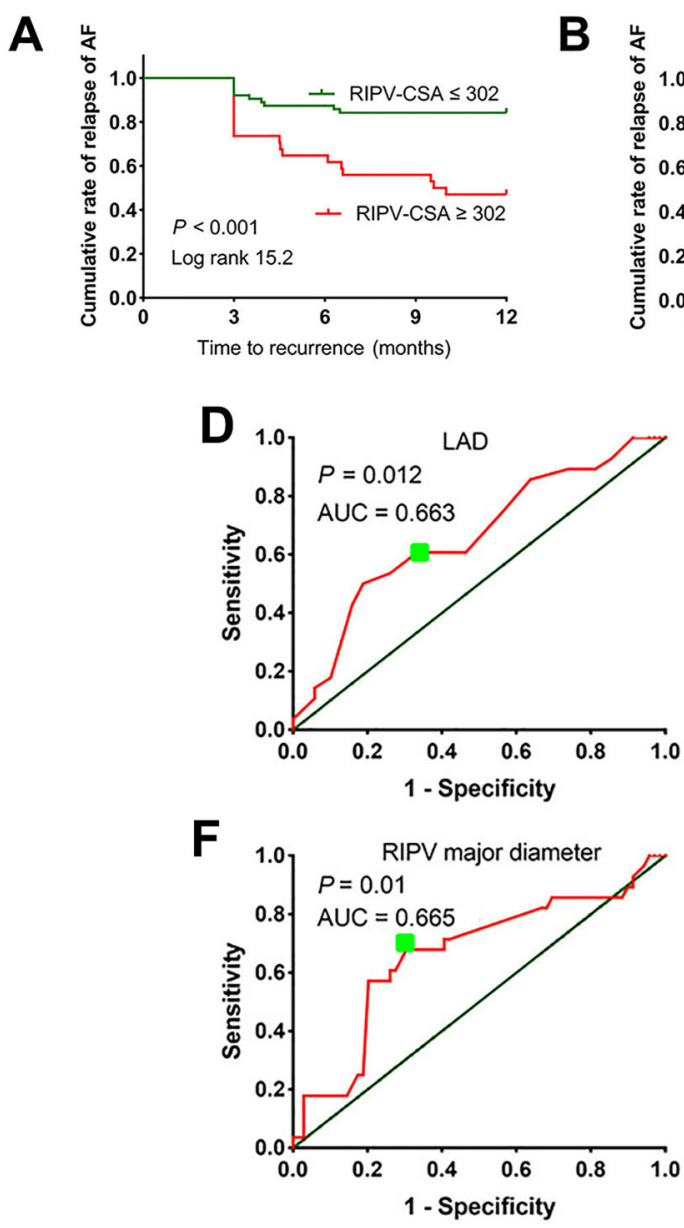

B

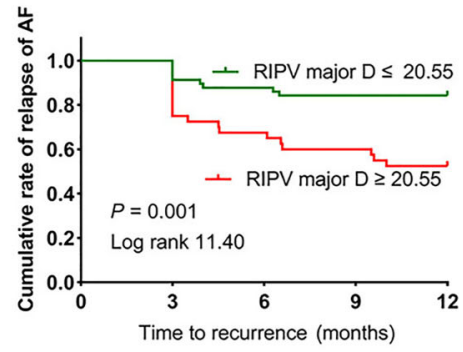

C

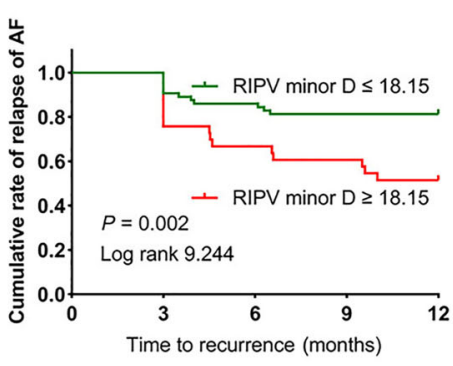

E

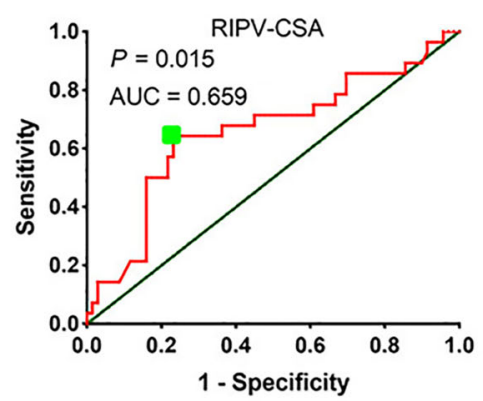

G

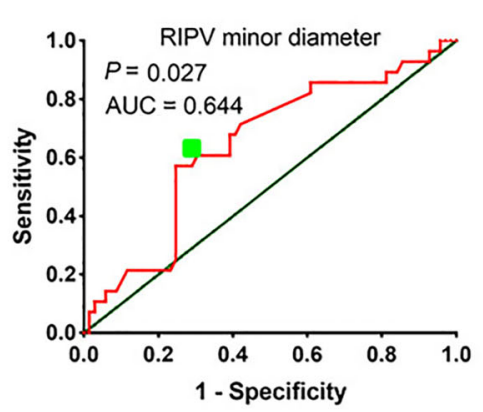

Figure 3. A-C, Cumulative proportional probability of atrial fibrillation (AF) recurrence in patients with a right inferior pulmonary vein (RIPV)-cross-sectional area (CSA) $\leqslant$ or $>302 \mathrm{~mm}^{2}$, a RIPV major diameter (D) $\leqslant$ or $>20.55 \mathrm{~mm}$, and a RIPV minor D $\leqslant$ or $>18.15$ $\mathrm{mm}$. The optimized cutoff points were obtained from the receiver operating characteristic curves. D-G, Accuracies of left atrial diameter (LAD), RIPV-CSA, RIPV major diameter, and RIPV minor diameter for predicting AF recurrence are reported as areas under the receiver operating characteristic curves (AUC). 
rate, especially in patients with a short duration of recurrence or asymptomatic recurrent AF. In addition, long-term follow-up after ablation is needed to determine the actual recurrence rate.

\section{Conclusions}

The major diameter and CSA of the RIPV are independent risk factors that can predict $A F$ recurrence at least as accurately as LAD in patients undergoing secondgeneration cryoballoon ablation.

\section{References}

1. Chugh SS, Havmoeller R, Narayanan K, Singh D, Rienstra $M$, Benjamin EJ, et al. Worldwide epidemiology of atrial fibrillation: a Global Burden of Disease 2010 Study. Circulation 2014; 129: 837-847, doi: 10.1161/CIRCULATIONAHA. 113.005119 .

2. Kirchhof P, Benussi S, Kotecha D, Ahlsson A, Atar D, Casadei B, et al. 2016 ESC Guidelines for the management of atrial fibrillation developed in collaboration with EACTS. Eur Heart $J$ 2016; 37: 2893-2962, doi: 10.1093/eurheartj/ehw210.

3. Heeger $\mathrm{CH}$, Wissner E, Wohlmuth P, Mathew S, Hayashi K, Sohns C, et al. Bonus-freeze: benefit or risk? Two-year outcome and procedural comparison of a "bonus-freeze" and "no bonus-freeze" protocol using the second-generation cryoballoon for pulmonary vein isolation. Clin Res Cardiol 2016; 105: 774-782, doi: 10.1007/s00392-016-0987-8.

4. Furnkranz A, Bordignon S, Dugo D, Perotta L, Gunawardene $M$, Schulte-Hahn B, et al. Improved 1-year clinical success rate of pulmonary vein isolation with the secondgeneration cryoballoon in patients with paroxysmal atrial fibrillation. J Cardiovasc Electrophysiol 2014; 25: 840-844, doi: $10.1111 /$ jce.12417.

5. Akkaya E, Berkowitsch A, Zaltsberg S, Greiss H, Hamm $\mathrm{CW}$, Sperzel J, et al. Second-generation cryoballoon ablation as a first-line treatment of symptomatic atrial fibrillation: two-year outcome and predictors of recurrence after a single procedure. Int J Cardiol 2018; 259: 76-81, doi: 10.1016/j. ijcard.2017.11.011.

6. Lemola K, Hall B, Cheung P, Good E, Han J, Tamirisa K, et al. Mechanisms of recurrent atrial fibrillation after pulmonary vein isolation by segmental ostial ablation. Heart Rhythm 2004; 1: 197-202, doi: 10.1016/j.hrthm.2004.03.071.

7. Ouyang F, Antz M, Ernst S, Hachiya H, Mavrakis H, Deger $\mathrm{FT}$, et al. Recovered pulmonary vein conduction as a dominant factor for recurrent atrial tachyarrhythmias after complete circular isolation of the pulmonary veins: lessons from double Lasso technique. Circulation 2005; 111: 127-135, doi: 10.1161/ 01.CIR.0000151289.73085.36.

8. Calkins H, Hindricks G, Cappato R, Kim YH, Saad EB, Aguinaga L, et al. $2017 \mathrm{HRS} / E H R A / E C A S / A P H R S / S O L A E C E$ expert consensus statement on catheter and surgical ablation of atrial fibrillation. Europace 2018; 20: e1-e160, doi: 10.1093/europace/eux274.

9. Tsyganov A, Petru J, Skoda J, Sediva L, Hala P, Weichet J, et al. Anatomical predictors for successful pulmonary vein

\section{Supplementary Material}

Click here to view [pdf]

\section{Acknowledgments}

We are grateful to Xi'an Jiaotong University Health Science Center for their technical assistance. This research was supported by the Clinical Research Award of the First Affiliated Hospital of Xi'an Jiaotong University, China (No. XJTU1AF-CRF-2015-007).

isolation using balloon-based technologies in atrial fibrillation. J Interv Card Electrophysiol 2015; 44: 265-271, doi: 10.1007/s10840-015-0068-3.

10. Guler E, Guler GB, Demir GG, Kizilirmak F, Gunes HM, Barutcu I, et al. Effect of pulmonary vein anatomy and pulmonary vein diameters on outcome of cryoballoon catheter ablation for atrial fibrillation. Pacing Clin Electrophysiol 2015; 38: 989-996, doi: 10.1111/pace.12660.

11. Hauser TH, Essebag V, Baldessin F, McClennen S, Yeon $\mathrm{SB}$, Manning WJ, et al. Prognostic value of pulmonary vein size in prediction of atrial fibrillation recurrence after pulmonary vein isolation: a cardiovascular magnetic resonance study. J Cardiovasc Magn Reson 2015; 17: 49, doi: 10.1186/ s12968-015-0151-z.

12. Nedios S, Tang M, Roser M, Solowjowa N, Gerds-Li JH, Fleck $\mathrm{E}$, et al. Characteristic changes of volume and threedimensional structure of the left atrium in different forms of atrial fibrillation: predictive value after ablative treatment. J Interv Card Electrophysiol 2011; 32: 87-94, doi: 10.1007/ s10840-011-9591-z.

13. January CT, Wann LS, Alpert JS, Calkins H, Cigarroa JE, Cleveland JC, Jr., et al. 2014 AHA/ACC/HRS guideline for the management of patients with atrial fibrillation: a report of the American College of Cardiology/American Heart Association Task Force on practice guidelines and the Heart Rhythm Society. Circulation 2014; 130: e199-267, doi: 10.1161/CIR.0000000000000041.

14. Kim YH, Marom EM, Herndon JE 2nd, McAdams HP. Pulmonary vein diameter, cross-sectional area, and shape: CT analysis. Radiology 2005; 235: 43-49; discussion 49-50, doi: 10.1148/radiol.2351032106.

15. Gottdiener JS, Bednarz J, Devereux R, Gardin J, Klein A, Manning WJ, et al. American Society of Echocardiography recommendations for use of echocardiography in clinical trials. J Am Soc Echocardiogr 2004; 17: 1086-1119, doi: 10.1016/j.echo.2004.07.013.

16. Pencina MJ, D’Agostino RB Sr, D’Agostino RB Jr, Vasan RS. Evaluating the added predictive ability of a new marker: from area under the ROC curve to reclassification and beyond. Stat Med 2008; 27: 157-172; discussion 207-212, doi: 10.1002/sim.2929.

17. Hassink RJ, Aretz HT, Ruskin J, Keane D. Morphology of atrial myocardium in human pulmonary veins: a postmortem analysis in patients with and without atrial fibrillation. 
J Am Coll Cardiol 2003; 42: 1108-1114, doi: 10.1016/S07351097(03)00918-5

18. Haissaguerre $M$, Jais $P$, Shah DC, Takahashi $A$, Hocini $M$, Quiniou G, et al. Spontaneous initiation of atrial fibrillation by ectopic beats originating in the pulmonary veins. $N$ Engl $J$ Med 1998; 339: 659-666, doi: 10.1056/NEJM199809033 391003.

19. Andrade JG, Khairy P, Macle L, Packer DL, Lehmann JW, Holcomb RG, et al. Incidence and significance of early recurrences of atrial fibrillation after cryoballoon ablation: insights from the multicenter Sustained Treatment of Paroxysmal Atrial Fibrillation (STOP AF) Trial. Circ Arrhythm Electrophysiol 2014; 7: 69-75, doi: 10.1161/CIRCEP.113. 000586.

20. Shimamoto K, Miura F, Shimatani Y, Nishioka K, Inoue I. Pulmonary vein volume predicts the outcome of radiofrequency catheter ablation of paroxysmal atrial fibrillation. PLoS One 2018; 13: e0201199, doi: 10.1371/journal.pone. 0201199.

21. Peters DC, Wylie JV, Hauser TH, Nezafat R, Han Y, Woo JJ, et al. Recurrence of atrial fibrillation correlates with the extent of post-procedural late gadolinium enhancement: a pilot study. JACC Cardiovasc Imaging 2009; 2: 308-316, doi: 10.1016/j.jcmg.2008.10.016.

22. Gerstenfeld EP, Callans DJ, Dixit S, Zado E, Marchlinski FE. Incidence and location of focal atrial fibrillation triggers in patients undergoing repeat pulmonary vein isolation: implications for ablation strategies. J Cardiovasc Electrophysiol 2003; 14: 685-690, doi: 10.1046/j.1540-8167.2003.03013.x.

23. Mainigi SK, Sauer WH, Cooper JM, Dixit S, Gerstenfeld EP, Callans DJ, et al. Incidence and predictors of very late recurrence of atrial fibrillation after ablation. $J$ Cardiovasc Electrophysiol 2007; 18: 69-74, doi: 10.1111/j.1540-8167.2006. 00646.x.

24. Shah AN, Mittal S, Sichrovsky TC, Cotiga D, Arshad A, Maleki $\mathrm{K}$, et al. Long-term outcome following successful pulmonary vein isolation: pattern and prediction of very late recurrence. J Cardiovasc Electrophysiol 2008; 19: 661-667, doi: 10.1111/j.1540-8167.2008.01101.x

25. den Uijl DW, Tops LF, Delgado V, Schuijf JD, Kroft LJ, de Roos $\mathrm{A}$, et al. Effect of pulmonary vein anatomy and left atrial dimensions on outcome of circumferential radiofrequency catheter ablation for atrial fibrillation. Am J Cardiol 2011; 107: 243-249, doi: 10.1016/j.amjcard.2010.08.069

26. Hu QM, Li Y, Xu CL, Han J, Zhang HB, Han W, et al. Analysis of risk factors for recurrence after video-assisted pulmonary vein isolation of lone atrial fibrillation--results of 5 years of follow-up. J Thorac Cardiovasc Surg 2014; 148: 2174-2180, doi: 10.1016/j.jtcvs.2013.10.054.

27. Balk EM, Garlitski AC, Alsheikh-Ali AA, Terasawa T, Chung $\mathrm{M}$, Ip S: Predictors of atrial fibrillation recurrence after radiofrequency catheter ablation: a systematic review. J Cardiovasc Electrophysiol 2010; 21: 1208-1216, doi: 10.1111/ j.1540-8167.2010.01798.x.

28. Tsao HM, Yu WC, Cheng HC, Wu MH, Tai CT, Lin WS, et al. Pulmonary vein dilation in patients with atrial fibrillation: detection by magnetic resonance imaging. $J$ Cardiovasc Electrophysiol 2001; 12: 809-813, doi: 10.1046/j.15408167.2001.00809.x

29. Chen WT, Chang SL, Lin YJ, Lo LW, Hu YF, Chao TF, et al. The impact of anatomical remodeling of the left atrium and pulmonary vein on the recurrence of paroxysmal atrial fibrillation after catheter ablation. Int J Cardiol 2014; 176: 1173-1175, doi: 10.1016/j.ijcard.2014.07.250.

30. Habibi M, Lima JA, Khurram IM, Zimmerman SL, Zipunnikov $\mathrm{V}$, Fukumoto $\mathrm{K}$, et al. Association of left atrial function and left atrial enhancement in patients with atrial fibrillation: cardiac magnetic resonance study. Circ Cardiovasc Imaging 2015; 8: e002769, doi: 10.1161/CIRCIMAGING.114.002769.

31. Nedios S, Koutalas E, Kosiuk J, Sommer P, Arya A, Richter $S$, et al. Impact of asymmetrical dilatation of the left atrium on the long-term success after catheter ablation of atrial fibrillation. Int J Cardiol 2015; 184: 315-317, doi: 10.1016/j. ijcard.2015.02.078.

32. Bisbal F, Alarcon F, Ferrero-de-Loma-Osorio A, GonzalezFerrer JJ, Alonso C, Pachon M, et al. Left atrial geometry and outcome of atrial fibrillation ablation: results from the multicentre LAGO-AF study. Eur Heart J Cardiovasc Imaging 2018; 19: 1002-1009, doi: 10.1093/ehjci/jey060.

33. Varela M, Bisbal F, Zacur E, Berruezo A, Aslanidi OV, Mont $L$, et al. Novel computational analysis of left atrial anatomy improves prediction of atrial fibrillation recurrence after ablation. Front Physiol 2017; 8: 68, doi: 10.3389/fphys.2017. 00068. 